Extraction à l'éther de pétrole.

\begin{tabular}{|c|c|c|c|}
\hline Insoluble dans l'aleool (céphaline) & 3,67 & 1,52 & $1 / 1,0$ \\
\hline Soluble dans l'alcool (lécithine) ... & 2,67 & 1,11 & $1 / 0,9$ \\
\hline Soluble à chaud, insoluble à froid ..... & 3,20 & 1,35 & $1 / 1,1$ \\
\hline \multicolumn{4}{|l|}{ ctraction à l'alcool-benzol. } \\
\hline Insoluble dans l'aleool (eéphaline) & 2,69 & 1,21 & $1 / 1,0$ \\
\hline Soluble dans l'alcool (lécithine) .. & 2,38 & 1,20 & $1 / 1,1$ \\
\hline Soluble à chaud, insoluble à froid ..... & - & - & - \\
\hline
\end{tabular}

La plupart des phosphatides n'étaient pas pures ; une céphaline pure a environ $4,1 \%$ de $\mathrm{P}$ et une lécithine pure $3,8 \%$ de $\mathrm{P}$. Il semble donc que le chauffage ait eu quelque influence; toutes les phosphatides étaient de couleur très sombre et il est possible que quelques petites quantités de sucre ou de sucre brûlé soient présentes. Il ést très ditficile d'éliminer les dernières traces des sous-produits. D'un autre côté, il est remarquable que le rapport $\mathrm{P} / \mathrm{N}$ ait été dans tous les cas exactement ou presque exactement $1 / 1$, ce qui signifie que toutes les phosphatides séparées sont des monoaminomonophosphatides.

Ces chiffres correspondent à ceux d'une étude précédente (1) dans laquelle est donnée également une revue des études précédentes sur les phosphatides du lait. On avait extrait du lait sec les phosphatides avec seulement de l'alcool et de l'alcool et du benzol. La proportion de lécithine était de $58,3 \%$, celle de céphaline : $41,7 \%$. On n'avait pas procédé à d'autres séparations.

Il ressort de ces recherches que les phosphatides existent aussi dans le lait et dans le beurre sous deux formes différentes, sous forme de phosphatides "libres", et de phosphatides "liées»; que dans chaque cas il existe au moins trois types différents de phosphatides; et que toutes les phosphatides extraites sont du type monoaminomonophosphatide. Il doit être mentionné que la matière première, le résidu de beurre, peut avoir subi une certaine modification qui peut avoir influencé jusqu'à un certain point la composition des phosphatides.

\title{
LE DOSAGE DE LA MATIÈRE GRASSE DANS LES FROMAGES (2)
}

\section{par DANIEL FLORENTIN}

Ce sujet paraît, a priori, complètement épuisé, tout ayant été dit sur la question.

Tout récemment, M. PIen, dans la Revue Le Lait (t. XVII,

(1) B. REWALD. Le Lait, t. XVII, mars 1937, p. 225-229.

(2) Annales des Falsifications et des. Fraudeৎ, no 355-356, juillet-août 1938, p. 351. 
1937, p. 569), a passé en revue les différentes méthodes de dosage de la matière grasse dans les fromages, toutefois dans le but, comme il l'indique, de choisir une méthode industrielle rapide.

Notre but est assez différent, puisque nous visons à définir une méthode précise de dosage destinée aux experts et aux laboratoires officiels qui ont à examiner des fromages pour rechercher s'ils répondent bien aux spécifications du décret du 20 octobre 1936 (1) ; ce décret ayant fixé des limites minima, il est indispensable de disposer d'une méthode fidèle et ne prêtant à aucune critique.

Or, nous avons eu l'occasion de constater, après beaucoup d'autres, que les différents laboratoires de contrôle obtenaient des chiffres de matière grasse parfois variables; une revue de l'industrie fromagère n'indiquait-elle pas récemment que trois laboratoires officiels avaient obtenu pour un même fromage, les résultats suivants : $36,3,49,40,51,70 \%$ de matière grasse.

Pour ne parler que du Laboratoire municipal de Paris, nous y utilisions, jusqu'ici, deux méthodes : l'une est la méthode classique d'extraction à l'éther, après broyage du fromage sec avec du sable, l'autre est la méthode de Lézé (appliquée tout d'abord aux laits : C. R., t. CX, 1890 , p. 647) qui est relatée, en particulier dans le traité de G. Pellerin. Elle consiste à attaquer deux grammes de fromage par $30 \mathrm{~cm}^{3}$ d'acide chlorhydrique concentré au bainmarie ; après refroidissement, la masse est jetée sur le filtre, puis lavée jusqu'à neutralité ; le filtre est séché puis épuisé à l'éther ou à la benzine.

Ce procédé paraissait avantageux quand le fromage était dur et se malaxait mal avec le sable.

Faisons observer que le principe de cette méthode se retrouve dans la méthode d'expertise internationale de SснміDT-BondZYNSKy-RAtSlaff, dérivée de la méthode d'expertise de BondZYNSKY et adoptée par la Convention de Rome du 26 avril 1934. Dans cette méthode, le fromage est également désagrégé à l'aide d'acide chlorhydrique, mais la matière grasse est extraite directement de la liqueur à l'aide de solvants.

Enfin, dans la méthode de dosage de la matière grasse dans les laits en poudre de WEIBULL, on dissout également la caséine dans l'acide chlorhydrique. Or, nous avons constaté que les chiffres obtenus par les méthodes utilisant l'acide chlorhydrique pour la destruction de la caséine étaient toujours inférieurs à ceux que l'on obtenait avec les méthodes par épuisement direct.

En particulier, dans ma note relative au dosage de la matière

(1) Le Lait, t. XVII, 1937, pages 98 et 101. 
grasse dans les poudres de lait (Annales des Falsifications, 1932, p. 88), j'avais signalé que la méthode d'extraction au Soxhlet fournissait des résultats supérieurs à ceux obtenus avec la méthode de Werbull, sans d'ailleurs que j'explique à cette époque les causes de ces écarts.

A la réflexion, nous avons pensé que ce traitement brutal par un acide minéral démolissait plus ou moins les glycérides constituant le beurre et qu'ainsi une fraction de la matière grasse transformée en glycérine et acides, solubles dans l'eau, disparaissait.

C'est bien ee qui se produit en effet, ainsi qu'on le constate si on étudie en détail la réaction.

Pour simplifier la question, nous avons traité directement 10 grammes de beurre pur par $50 \mathrm{~cm}^{3}$ d'acide chlorhydrique $\mathrm{D}=1,125$, au voisinage de l'ébullition pendant 1 heure. Après refroidissement, la matière grasse a été séparée, puis la solution chlorhydrique soigneusement épuisée à l'éther; celui-ci est lavé, puis évaporé. On isole ainsi une petite quantité d'acides gras solubles dans l'eau (acide butyrique et autres). D'autre part, l'acide est évaporé dans le vide et le résidu, repris par l'alcool, permet d'isoler une petite quantité de glycérine.

Dans ce traitement, la perte de poids du beurre (anhydre) est d'environ $5 \%$.

D'autre part, la matière grasse avant et après traitement possède les caractéristiques suivantes :

\begin{tabular}{|c|c|c|}
\hline & Beurre initial & Beurre traité \\
\hline Indice Crismer $\ldots \ldots \ldots$ & $54^{\circ} 4$ & $32 \circ 2$ \\
\hline Acidité \% (en SO$\left.{ }^{4} \mathrm{H}^{2}\right)$ & $0 \mathrm{gr} .17 \mathrm{~s}$ & $1 \mathrm{gr} .837$ \\
\hline Indice de Reichert acidulé & 35,81 & 32,42 \\
\hline Acides volatils insolubles & 5,44 & 4,1 \\
\hline
\end{tabular}

Ces chiffres indiquent nettement que le traitement à l'acide chlorhydrique a altéré profondément la matière grasse du beurre ; une partie des glycérides s'est scindée en acides gras et glycérine. La glycérine étant soluble dans l'eau ainsi qu'une partie des acides gras, il en résulte une perte de poids assez importante de la matière grasse.

Ceci explique pourquoi les méthodes basées sur l'emploi de l'acide chlorhydrique fournissent toujours des résultats trop faibles, qu'il s'agisse des laits en poudre (méthode de WErBuLL) ou des fromages.

Mais la méthode internationale qui utilise l'acide chlorhydrique de $\mathrm{D}=1,125$ pour désagréger le fromage, échappe-t-elle à ces critiques ?

Pour trancher expérimentalement la question, nous avons 
analysé, avec un grand soin, un même échantillon de fromage (Port-Salut) par la méthode au sable, la méthode de LEzÉ, et la méthode internationale. Voici nos résultats :

Méthode au sable Méthode Lezé Méthode internationale

Matière grasse :

$\begin{array}{llll}1) & 45,5 & 44,5 & 44,9 \\ 2) & 45,4 & 44,8 & 44,8\end{array}$

Examen de la matière grasse (acidité exprimée en acide oléique) :
1)
6 gr. 45
$12 \mathrm{gr} .1$
$9 \mathrm{gr} .62$
2)
7 gr. 30
10 gr. 2
$8 \mathrm{gr} .40$

D'après ces résultats, on voit que la méthode de LEzÉ (attaque par $\mathrm{HCl}$ ) est nettement à rejeter, car elle fournit des résultats trop faibles et, encore, ceux-ci sont-ils parmi les meilleurs d'après nos expériences.

La méthode de Schmid-Bondzynski-Ratzlaff fournit également des résultats inférieurs à ceux de la méthode par extraction directe ; en outre, la matière obtenue est altérée ainsi qu'en témoigne son acidité,

Si on ajoute que la méthode internationale est d'une application longue et délicate, on ne voit vraiment pas pourquoi le Congrès de Rome l'a préférée au vieux procédé de Duchaux, tel qu'il est relaté dans ses "Principes de laiterie ».

Compte tenu des observations ci-dessus, nous nous permettons d'émettre le vœu qu'on revienne sur les conclusions de la Convention de Rome du 26 avril 1934, et qu'on utilise à l'avenir, comme méthode officielle pour le dosage de la matière grasse dans les fromages, la méthode dite "au sable" qui permet d'obtenir toute la matière grasse dans son état primitif, de telle sorte qu'il est loisible à l'expert de procéder à une étude dans le but de déterminer son adultération éventuelle.

Certains chimistes préfèrent utiliser, à la place du sable, le sulfate de sodium anhydre dont l'emploi est également fort commode.

Quoi qu'il en soit, M. PIEN a donné, dans l'article cité plus haut, une relation détaillée de la méthode "au sable " telle qu'il l'utilise dans son laboratoire.

Nous croyons cependant utile d'indiquer d'une façon assez détaillée le procédé que nous utilisons dans nos expertises.

Deux grammes de fromage (échantillon moyen, privé de sa croûte) sont coupés en menus fragments et disposés sur trente grammes environ de sable lavé et sec, placés dans une capsule et mis pendant 5 à 6 heures dans une étuve électrique portée à $100^{\circ}$. (Le procédé de DucLAux qui consistait à broyer le fromage avec le sable et à déterminer la perte d'eau à basse température $\left(50\right.$ à $60^{\circ}$ ) serait évidemment préférable, mais se prête mal au 
travail en grande série.) La perte de poids fournit la teneur en eau ; après pesée, le contenu de la capsule est broyé au mortier pendant 10 minutes, de façon à obtenir une masse homogène. Ce broyage effectué sur un fromage sec se réalise aisément. Le tout est placé dans un filtre à plis qui est disposé dans un petit appareil Soxhlet, la capsule et le mortier sont soigneusement lavés à l'éther qui est placé dans l'appareil Soxhlet.

Après quelques heures d'extraction, l'éther est évaporé et la fiole conique en verre Pyrex, qui reçoit le Soxhlet, et qui a été préalablement tarée, est pesée après un passage à l'étuve à $100^{\circ}$. On obtient ainsi le poids de toute la matière grasse contenue dans le fromage.

Une expérience ancienne nous permet d'affirmer que cette méthode très simple, et qui se prête au travail en série, fournit des résultats remarquablement concluants.

\section{REVUE}

\section{LE LANITAL (1)}

Le "lanital ", ce textile artificiel inventé en Italie, est à l'ordre du jour. La liste des pays ayant acquis lalicence Ferretti comprend actuellement : l'Italie, la Pologne, la France, la Belgique, l'Allemagne, l'Angleterre, le Canada, la Hollande et la Tchécoslovaquie.

Devant l'intérêt - et il faut le dire, les "réactions " diverses - que suscite cette matière, il nous a paru intéressant de compléter les informations déjà publiées dans notre revue, par une documentation beaucoup plus détaillée. Nous l'avons demandée à un spécialiste en lui laissant la liberté et la responsabilité de ses appréciations et de ses conclusions.

$$
\text { *** }
$$

En créant la rayonne, l'industrie textile a cherché un remplaçant à la soie naturelle, dont la production, pour des raisons diverses dont les principales relèvent du domaine agricole propre à la sériciculture, devenait de jour en jour plus rare pendant que la demande ne cessait de croître.

L'éternelle loi économique fit que cette matière, noble entre toutes, atteignit un prix tellement élevé qu'elle en devint un article de haut luxe réservé à une classe privilégiée de consommateurs.

De la rayonne coupée, est sortie la schappe artificielle, laquelle, filée sur les continus à coton ou sur les assortiments de laine, soit

(1) Le Nord textile, n० 935,2 avril 1938. 